\title{
Investigating a comorbid pattern of ill-health among late adolescents in relation to transdiagnostic processes: A prospective community study
}

\author{
Katja Boersma, Maria Tillfors \\ Örebro University, Örebro, Sweden; katja.boersma@,oru.se \\ Received 30 August 2013; revised 30 September 2013; accepted 15 October 2013 \\ Copyright (C) 2013 Katja Boersma, Maria Tillfors. This is an open access article distributed under the Creative Commons Attribution \\ License, which permits unrestricted use, distribution, and reproduction in any medium, provided the original work is properly cited.
}

\begin{abstract}
An increase in anxiety, depressed mood and sleep problems has been observed among adolescents. These symptoms have high rates of comorbidity and shared psychological processes have been proposed as maintaining factors. This study examined the occurrence and development of individual profiles of depressive symptoms, anxiety and sleeps problems and investigated them in relation to catastrophic thinking and cognitive avoidance. We used longitudinal data from a community sample of 379 senior high school students at two time points, one year apart. Five clusters were identified: a low scores cluster, a sleep problems cluster, a comorbidity cluster (high on all variables), a low mood cluster and a cluster with elevation on anxiety and depressed mood ("distress"). In general, the clusters showed stability across time. However, for the low mood and "distress" cluster, there was also an increased odds of developing sleep problems. The comorbidity and the "distress" cluster displayed the highest levels of catastrophic thinking. In conclusion, symptom patterns differed among adolescents and were stable over time. Anxiety and/or depressive symptoms were a risk factor for the development of sleep problems. Symptom constellations were related to differences in catastrophic thinking and cognitive avoidance and this may explain maintenance and exacerbation of problems over time.
\end{abstract}

Keywords: Mental Health; Adolescents; Comorbidity; Transdiagnostics; Cognitive Processes

\section{INTRODUCTION}

Several studies have shown that there is an increasing prevalence of symptoms of anxiety, depressed mood, and sleep problems among adolescents in Sweden during the past decennia [1]. A similar trend has been shown in other post-industrial countries (e.g. [2,3]). This increase in prevalence mirrors society at large but is most pronounced within the age span $16-24$ [1]. As with adult psychological health, adolescent girls show a markedly higher prevalence of problems than boys, although the trend of increase is similar for boys and girls [4]. Hence, adolescence can be seen as the point of departure for development of psychological and health related problems later on in life. Although we might expect these problems to disappear with maturation, evidence suggests that they do not; instead, the majority of adolescents who suffer from mental health problems, continue to suffer as adults, or develop even more serious problems (e.g. [5,6]). Also, mental health problems during adolescence are a risk factor for a range of negative outcomes such as for example school dropout [7].

In summary, evidence shows that adolescence is a period of high susceptibility for mental health problems and that the number of adolescents with mental health problems has increased during the past decades. Adolescence is the point of departure for many mental health problems and these may lead to a wide range of negative outcomes both for the individual and for society. Therefore, it is important to increase understanding of occurrence of common mental health problems in adolescents, since this would give the possibility to intervene early.

A high rate of comorbidity has been observed between mental health problems such as anxiety, depression and sleep problems in both the general population and in clinical populations. Not in the least in clinical settings, the co-occurrence of these symptom constellations is the rule rather than the exception [8]. If a person has multi- 
ple mental health problems, this often means more severe pathology as well as a higher degree of disability. Hence, it is important to gain insight into how and why these symptom-constellations co-occur.

In light of the high level of comorbidity of psychological problems, it is also of interest to examine the order in which symptoms of anxiety, depression and sleep problems arise. Previous research shows a progressive developmental pattern that often starts with symptoms of anxiety [9]. These results indicate that anxiety may be a risk factor for developing depression and sleep problems. This is partly in line with the traditional view that sleep problems are a consequence of anxiety and depression (e.g. [10]). However, it also appears that the reverse could be the case, i.e., that sleep problems in youth can lead to increased risk of developing both anxiety (e.g. [11]) and depression [12]. One possible mechanism for the latter may be that sleep deprivation has proven to cause difficulties in emotion regulation [13]. Hence, the relationship between anxiety problems, depressive symptoms and sleep problems seems to be complex and needs to be explored further, in longitudinal studies.

The high rates of comorbidity observed between psychological problems could partly be explained by the shared psychological processes that characterize them [8]. In this "transdiagnostic" perspective, the focus is on identifying psychological factors that can be observed across diagnostic boundaries. It is proposed that these shared factors serve to maintain ill-health. If shown to be true, this may have considerable implications for our understanding of the development of psychological problems, but also for developing effective and parsimonious interventions. The transdiagnostic perspective has mostly focused on cognitive and behavioral factors maintaining psychological problems. Specifically, psychological problems seem to be maintained by cognitive and behavioral processes that function as strategies to regulate negative emotion. Prominent examples are avoidant behaviors on an overt level (e.g., avoiding an anxiety provoking situation) and on a covert level (e.g., try to suppress or distract from intense negative thoughts), as well as repetitive negative thinking [8]. Repetitive negative thinking is the generic umbrella term capturing thought processes such as worry, rumination and catastrophic thinking [14]. These thought processes have important commonalities in that they are all negative, repetitive, abstract, passive and relatively uncontrollable in nature $([8,14])$. It has been proposed that repetitive negative thinking, by means of its abstract and predominantly verbal nature, may function to reduce both aversive imagery and unpleasant physical anxiety sensations related to potential problems (e.g. [15]). Thus, one of the proposed functions of repetitive negative thinking may be avoided of unpleasant inner affective experiences. Inadvertently how- ever, the consequence is assumed to be a reduction in long term effective emotional processing [14]. Given the proposed function of repetitive negative thinking, it might thus also be categorized as a form of covert avoidance.

In summary, both overt and covert avoidance could be seen as emotional control strategies that may work in the short term to reduce unpleasant inner experiences, but in the long term may become dysfunctional ways to regulate emotions. Hence, it is hypothesized that these behavioral and mental control strategies set in motion a negative vicious cycle that could explain the perpetuation of ill-health.

Indeed, control strategies like overt and covert avoidance have repeatedly been shown to be associated with maintenance of anxiety problems, depressed mood as well as sleep problems [8]. However, since the factors that maintain a problem are not necessarily the same as those that once caused it, a major question is in what way these transdiagnostic processes are involved in the development of mental ill-health.

Research on prevalence, etiology and treatment of psychological disorders is almost exclusively based on categorical diagnoses according to the DSM system [8]. One consequence is that the generalizability of such studies is restricted to more extreme, clinical, symptom levels and on quite specific symptom constellations based on relatively arbitrary "cut-offs". This may not reflect the dimensionality of mental health problems and may hinder the possibility to capture a comprehensive picture of the development of symptoms and of symptom comorbidity. Moreover, it is of great importance to investigate which mechanisms increase or decrease the risk for moving up- or downwards along the continuum.

To summarize, longitudinal studies mapping several psychological symptoms and transdiagnostic processes, as well as studies tracking their longitudinal course during the adolescent age period are needed to fill the gap of missing knowledge. Therefore, the first objective of this study was to examine clusters with different individual profiles of depressive symptoms, symptoms of anxiety, and sleep problems. We used longitudinal data from a community sample of adolescents in senior high school at two time points, one year apart. Furthermore, we examined the symptom constellations cross-sectional in relation to transdiagnostic processes at wave 1 , as well as investigated the moderating effect of gender. Specifically, we focused on two mental control strategies that could be categorized as forms of covert avoidance in dealing with unpleasant symptoms: "catastrophic thinking" (having thoughts that signal highly negative symptoms interpretations) and "cognitive avoidance" (having beliefs that signal that mental strain or emotionally charged situations should be avoided). We expected to 
find a subgroup of adolescents with a comorbid pattern of symptoms of anxiety, depressive symptoms and sleep problems that in a high degree display dysfunctional mental control strategies. Finally, we examined the stability of symptom constellations within individuals across a one year time period, and related symptom changes to mental control strategies over time.

Analytically, we used both a variable-oriented perspective and a person-oriented perspective in this study. A person-oriented perspective [16] will be used both to classify adolescents into different profiles of depressive symptoms, symptoms of anxiety, and sleep problems and to study change and stability of individual symptom constellations over time. An advantage of this approach is that it allows for a mapping of the development and cooccurrence of symptoms across time on an individual instead of on a variable level, and that it highlights possible interindividual differences in development (for a discussion of advantages of person-oriented approaches in the context of developmental psychology see for example [17-19]). It also provides the opportunity to study the comorbidity of symptoms that some individuals may experience in relation to the characteristics of hypothetical subgroups of individuals that have singular or no symptoms. We further use traditional variable-oriented statistical analyses to study the predictive validity of transdiagnostic variables as well as to validate and describe the subgroups.

\section{METHOD}

\subsection{Design}

This is a prospective study with two measurement points, baseline ("wave 1"), and 1-year follow-up ("wave $2 ")$.

\subsection{Participants and Procedure}

The participants were senior high school students from one school in a city (a total population of 23,400 inhabitants) in Western Europe (grades 10 through 12, age range between 16 and 20). This study is part of a three year longitudinal study regarding physical and psychological well-being in youths. The first data collection took place during the 2006-2007 school years. The following two waves of data were collected respectively during the 2007-2008 and the 2008-2009 school years. At each wave around $80 \%$ of all high school students participated.

The target sample included 1389 high school students from wave 1 and 2. Of these, 379 (31\%) students (54\% girls and $46 \%$ boys) were eligible for analyses. The inclusion criterium was that students had participated in both measurement points with complete data on the target variables. This criterion excluded 969 students who were either in their $3^{\text {rd }}$ year at the first measurement point (and therefore had graduated from high school at the second measurement point), or who were in their $1^{\text {st }}$ year at the second measurement point, or lastly who were absent from school the day the data collection took place. Due to a mishap in questionnaire distribution that resulted in incomplete data on the target variable sleep, an additional 41 students were excluded from the sample. As compared to the study sample, there was a significantly higher degree of boys in this group $\left(\chi_{2}(1)=22,11\right.$, $\mathrm{p}<0.001)$. However, there we no significant differences between this group and the study sample on level of anxious symptoms, depressive symptoms, or on ratings on a statement about sleep quality ("I sleep well"; $1=$ not at all true, $2=$ somewhat true, $3=$ moderately true, $4=$ very much true, and $5=$ completely true).

The students were recruited during school hours and they were informed that the participation was voluntary and confidential. In the $10^{\text {th }}$ grade questionnaires were administered by the school nurse and completed in class during regular school hours. The collection of questionnaires in the $11^{\text {th }}$ and the $12^{\text {th }}$ grades was administered by the teachers during the students' mentor time. Students were informed to put the questionnaire in separate envelopes in order to secure confidentiality. Students were not paid for participation. At the outset of the study, 70\% of the students lived with both biological parents, and $22 \%$ lived in single parent households. The unemployment rate in the community was $9 \%$ (Swedish average was $7.5 \%$ ); mean incomes were about $8 \%$ lower than that in the rest of the country. Most participants (92\%) were born in Sweden with at least one biological parent who was born in Sweden (91\%).

\subsection{Measures}

The following questionnaires/items were included in this study:

Depressive Symptoms. In order to assess symptoms of depressed mood, the "depressed mood" subscale of The Center for Epidemiologic Studies Depression Scale for Children (CES-DC; [20]) was used. In their study Olsson and von Knorring [20] conducted a factor analysis where four factors were extracted (explaining 58.9\% of the variance), reflecting depressed mood (Factor 1), tiredness and inability to concentrate and work (Factor 2), feelings of being outside and lonely (Factor 3), and lastly positively stated items (Factor 4). In this study we included the factor "depressed mood" which consisted of six items (Bothered, Blues, Depressed, Fearful, Crying and Sad). These items were chosen because this first factor explained the largest amount of variance and because it captures the core of depression, that is, low mood. The response scale varied from 0 to 3 , with the following verbal descriptors for the end points: $0=$ not at all, $3=$ often. A total score for the subscale was calculated by 
summing the ratings for the 6 items with higher scores indicating more depressed mood $(\operatorname{Min} / \mathrm{Max}=0 / 18)$. The Cronbach's alphas were $\alpha=0.86$ for Wave 1 and $\alpha=$ 0.87 for Wave 2 .

Anxiety symptoms. In order to asses symptoms of anxiety, the Anxiety subscale of the Hospital Anxiety and Depression Scale (HADS; [21] was used. The anxiety subscale consisted of 7 items e.g. "I feel tense or wound up" (anxiety). Subjects score each item on a 4point scale $(0-3)$ with end points varying with the item. A total score for the subscale was calculated by summing the ratings for the 7 items with higher scores indicating more anxiety $(\operatorname{Min} / \operatorname{Max}=0 / 21)$. The scale has, in reviews, as well as in the Swedish population specifically, been found to have satisfactory reliability (mean chronbach's alpha $\alpha=0.83)$ and construct validity ([22,23]). The Cronbach's alphas were $\alpha=0.75$ for Wave 1 and $\alpha=$ 0.78 for Wave 2 .

Sleep problems. In order to asses varying degrees of sleep problems, four questions were used, based on questions from the Basic Nordic Sleep Questionnaire [24] and the Uppsala Sleep Inventory [25]. A single item asked whether sleep problems had occurred during the past three months, answered categorically by a yes or no [26]. In addition, the severity of sleep difficulties was assessed by items allowing the detection of self-reported insomnia in line with the Diagnostic and Statistical manual of Mental Disorders criteria: sleep problem frequency of three nights or more per week, and difficulty initiating (sleep latency of 30 minutes or more) or maintaining sleep (30 minutes or more when awake during night) [27]. Using these questions, a pseudo scale consisting of varying degrees of sleep problems was created as follows: 0. Good sleep ("no" answer on have you had sleep problems during the past three months an no sleep latency or sleep maintenance difficulties). 1. Normal sleep ("no" answer on have you had sleep problems during the past three months, but allowing for sleep latency or sleep maintenance difficulties). 2. Insomnia symptoms (as insomnia but not occurring more than 3 times a week). 3. Insomnia (sleep problem at least three times a week during the past three months, plus at least 30 minutes sleep latency or sleep maintenance problems).

Cognitive Processes. In order to assess the use of mental control strategies in reaction to symptoms, the "Avoidant Safety Behaviors of a cognitive orientation" and the "Catastrophizing" subscales of the Safety Behaviors and Catastrophizing Scale (SBCS) were used [28]. The "Avoidant Safety Behaviors of a cognitive orientation" subscale has 4 items (example item: "I should avoid emotionally charged situations") and the "Catastrophizing" subscale has 6 items (example item: "I wonder if there is something seriously wrong with me"). Responses on all items were scored on an ordered re- sponse scale with the following verbal descriptors: $0=$ not at all true for me, $1=$ somewhat true for me, $2=$ moderately true for me, $3=$ very much true for me and 4 $=$ extremely true for me $(\mathrm{Min} / \mathrm{Max}$ respectively $0 / 16$ and 0/24). Throughout the manuscript these scales will be called respectively: "cognitive avoidance", and "catastrophic thinking". Previous investigations have found the scale to possess good psychometric properties $[28,29]$. The Cronbach's alphas for the scales at Wave 1 were "cognitive avoidance" $\alpha=0.75$ and "catastrophic thinking" $\alpha=0.83$ at Wave 2 "cognitive avoidance" $\alpha=0.77$ and "catastrophic thinking" $\alpha=0.82$.

Satisfaction with life. To validate the relationship of patterns of symptoms with a relevant external criterion related to severity of problems we selected self-reported satisfaction with life. Participants were requested to relate to a $1-5$ scaled general statement "I am satisfied with life" $(1=$ not at all true, $2=$ somewhat true, $3=$ moderately true, $4=$ very much true, and $5=$ completely true).

School absenteeism. To validate the relationship of patterns of symptoms with relevant school related outcomes we selected school absenteeism. In order to assess the degree of school absenteeism during the past three months, a 1 - 5 scaled question "have you been absent a whole day from school during the past three months?" was used $(1=$ has not happened, $2=$ happened once, $3=$ happened $2-3$ times, $4=$ happened $4-10$ times, and $5=$ happened more than 10 times).

\subsection{Statistical Analysis}

First measures were summarized using descriptive statistics. Gender differences in scoring patterns on the main variables were investigated using t-tests except for the quasi scaled sleep problems variable where MannWhitney U-test was used. There after cluster analyses were used to extract groups of individuals with similar scoring patterns on depressive symptoms, anxiety symptoms and sleep problems. Cluster analysis requires variables to be scored on scales with similar range as not to give any of the variables undue weight. Therefore, depressive symptoms and anxiety were rescaled to form a so called quasi absolute scale [19] in parallel to the sleep problem scaling. This type of scaling gives a useful impression of the degree of problems and allows for comparison of previously incomparable variables. Table 1 gives an overview of the cut off values for the quasi absolute scales. These are, insofar possible, based on empirically derived cut-off scores found in the literature $([10,22,26,30])$. Note that, since we included one factor out of four from the CES-DC scale (reflecting depressed mood), we had no direct reference for depression cut-off scores to rely on. Therefore, we used reported percentile values for the total score as a guiding principle [20]. 
Table 1. Cut off values and corresponding values on a quasiabsolute scale for depressive and anxiety symptoms and sleep problems.

\begin{tabular}{cccc}
\hline Quasi scale & CES-DC & HADS & Sleep problems \\
\hline $0=$ no problem & $0-3$ & $0-7$ & Good sleep \\
$1=$ sign of problem & $4-8$ & $8-10$ & Normal sleep \\
$2=$ clear problem & $9-15$ & $11-14$ & Insomnia symptoms \\
$3=$ serious problem & $16-18$ & $15-21$ & Insomnia \\
\hline
\end{tabular}

Note: CES-DC = depressive symptoms, HADS = anxiety symptoms.

The cluster analyses were done within Statistical Package for Social Sciences (SPSS) as well as within SLEIPNER, a statistical package that is developed for pattern-oriented analyses [16]. The data were analyzed using the LICUR rationale (LInking of ClUsters after removal of a Residue; [17]). This procedure encompasses the following. First, the sample of 379 subjects was examined for multivariate outliers at baseline and follow up separately. This procedure identified no candidates with diverging patterns to be excluded from further analysis. Thereafter, two hierarchical cluster analyses were performed cross sectionally at baseline (wave 1) and at the 12 month follow up (wave 2); using squared Euclidean distance as the similarity measure and Ward's method to minimize within-cluster differences. To ensure reasonably homogeneous clusters, we selected cluster solutions that explained at least $67 \%$ of the total error sum of squares for further analysis [16]. Thereafter, k-means cluster analyses were used to fine-tune the results. This procedure aims to increase the homogeneity of the clusters by allowing cases to move to a better fitting cluster if this leads to a reduction in the error sum of squares of the cluster solution.

Then, these two separate cluster solutions, extracted from the same sample at two different time points, were linked across time using the "centroid" procedure to examine structural stability and the "exacon" procedure to examine individual stability. The "centroid" procedure assists in examining the structural similarity of cluster solutions by calculating the average squared Euclidean distance between the centroids of the most optimally matched clusters. The closer to zero the values, the more similar the clusters are considered to be [31]. The exacon procedure produces a contingency table of the two cluster solutions and provides the possibility to examine common pathways: "types" (an overrepresentation of units in a cell). It calculates, among other things, the probability of by chance finding a cell frequency as high/ low as the observed one, and can in this way highlight relationships between clusters (the typical individual progressions from one cluster to another over time).

To ensure the validity and robustness of the cluster solution, the results of the baseline cluster analysis were replicated on a random subset of the total data set. Specifically, a random subset of the total data set $(\mathrm{N}=150)$ was cluster analysed at baseline using the same procedure as above. The cluster results for the subsample were then compared to the solution from the total data set using the centroid procedure in SLEIPNER. In addition, to ensure cluster stability, a cluster analysis was also performed with weighted average linkage as the similarity measure.

Thereafter, the cluster solution at baseline were described and validated on gender, cognitive processes, life satisfaction and school absenteeism. Depending on scaling level, ANOVA, Kruskal Wallis or Chi-square was used to statistically test differences between clusters on these variables. To investigate the moderating role of gender in the relationship between the cluster solution and the mental control strategies (i.e., the cognitive processes), a gender by cluster solution factorial ANOVA was performed. As a last step, an exploratory analysis was performed investigating whether the developmental pathways of respectively stability, increase and decrease in symptom severity were accompanied by differential changes in cognitive processes. ANOVA was used to test for significant differential changes in catastrophizing and cognitive avoidance. For all ANOVAs, due to unequal sample sizes, Gabriel's (equal variances) and Games Howell's (unequal variances) were used as post hoc tests. For the Kruskal Wallis tests pairwise comparisons with Bonferroni corrections were used.

\section{RESULTS}

Table 2 displays descriptive statistics for the main variables on a group level. As can be seen, girls score significantly higher on all variables.

\subsection{Clusters of Depressive Symptoms, Anxiety and Sleep Problems}

Figure 1 displays five clusters with differential individual patterns of depressive symptoms, anxious symptoms and sleep problems at wave 1 . This 5-cluster solution is a result of combination of hierarchical and $\mathrm{k}$ means cluster analyses and explains $73 \%$ of the total error sum of squares. All clusters are expressed in zscores. As can be seen, one cluster appears with relatively low scores on all variables (cluster 1 "low scores" cluster, $\mathrm{N}=155 \%, 41 \%$ ). Also, a cluster appears mainly characterized by sleep problems (cluster 2, "sleep problems" cluster, $\mathrm{N}=87 \%, 23 \%$ ). The third cluster consists of individuals with high levels of depressive and anxious symptoms as well as sleep problems (cluster 3 , "comorbidity" cluster, $\mathrm{N}=27 \%, 7 \%$ ). The fourth cluster is characterized by depressive symptoms only (cluster 4 , "low 
Table 2. Descriptive statistics at baseline (wave 1) for the whole group, as well as boys and girls separate on depressive symptoms, anxiety, sleep problems and cognitive processes.

\begin{tabular}{cccccc}
\hline & Total & Girls & Boys & & \\
\hline & Mean (sd) & Mean (sd) & Mean (Sd) & df & t/U \\
\hline Depressive symptoms $(0-18)$ & $4.6(4.2)$ & $6.3(4.4)$ & $2.6(3.0)$ & 358.2 & $9.9^{* * *}$ \\
Anxious symptoms $(0-21)$ & $5.1(3.5)$ & $6.1(3.8)$ & $4.0(2.7)$ & 363.2 & $6.3^{* * *}$ \\
Sleep problems $(0-3)$ & $1.2(0.7)$ & $1.3(0.8)$ & $1.1(0.7)$ & 376.2 & $15598.0^{*}$ \\
Catastrophizing $(0-24)$ & $15.7(1.9)$ & $17.1(5.1)$ & $14.1(4.4)$ & 359 & $5.9^{* * *}$ \\
Cognitive avoidance $(0-16)$ & $10.2(3.3)$ & $10.6(3.4)$ & $9.7(3.2)$ & 358 & $2.4^{*}$ \\
\hline
\end{tabular}

${ }^{*} \mathrm{p}<0.05 ;{ }^{* * *} \mathrm{p}<0.001 ; \mathrm{t}=$ test statistic for t-test, $\mathrm{U}=$ test statistic for Mann-Whitney test.

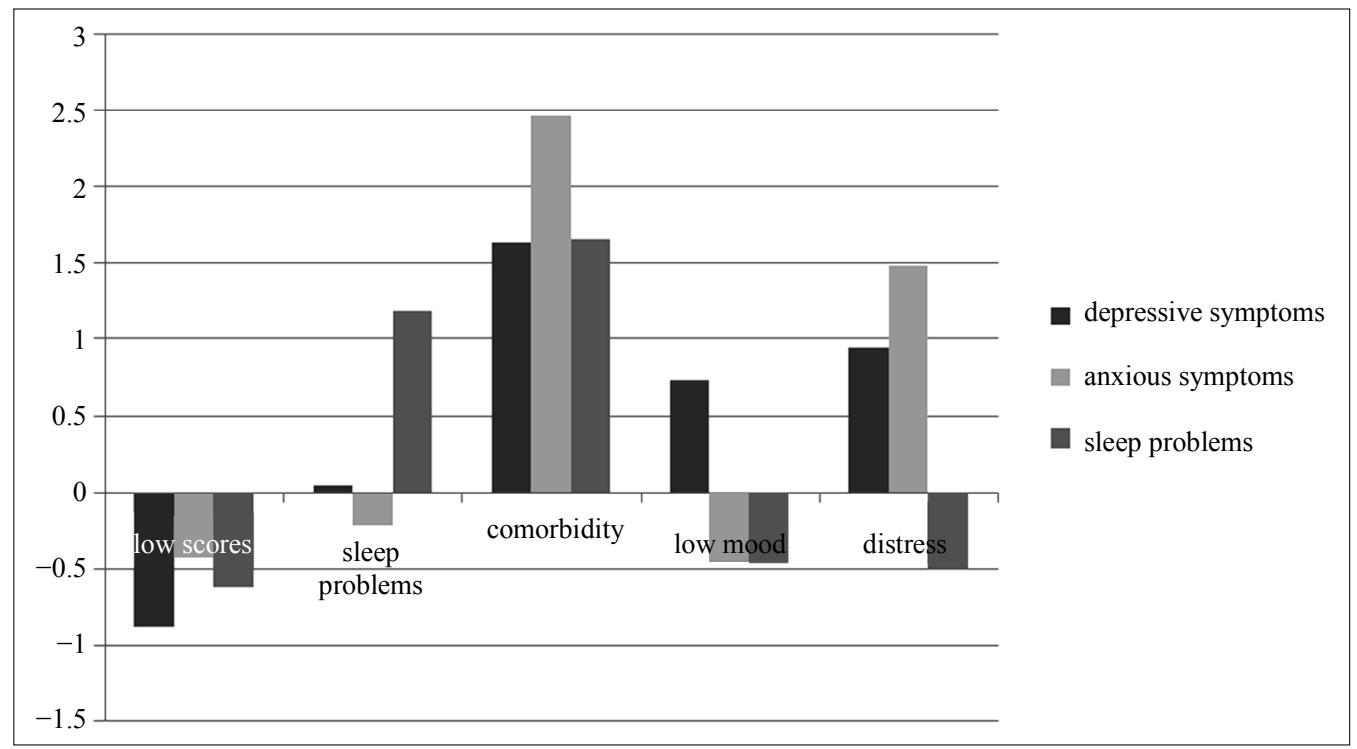

Figure 1. Baseline cluster solution using variables depressive symptoms, anxious symptoms and sleep problems rescaled on a quasi absolute scale. Note: Clusters are expressed on z-scores.

mood" cluster, $\mathrm{N}=75 \%, 20 \%$ ) and finally the fifth cluster by depressive as well as anxious symptoms (cluster 5, "distress" cluster, $\mathrm{N}=35 \%, 9 \%$ ).

\subsection{Validation of the Clustering Procedure}

To ensure that the cluster solution is not a mere product of random results, we compared a five cluster solution for a random subsample of the total data set $(\mathrm{N}=$ 150), with the five cluster solution for the total sample $(\mathrm{N}=379)$ using the centroid procedure in SLEIPNER. The centroid procedure calculates the average squared Euclidean distance (ASEDs) between the centroids of the two clusters that show closest resemblance. The results show that the ASEDs associated with these matches ranged from 0.003 to 0.25 with a mean of 0.099 . This represents a good agreement between the solutions. The percentage of explained error sums of squares in the new analysis on the subset was $72 \%$ (as compared to $73 \%$ for the total sample). To further ensure the stability of the cluster solution, a cluster analysis was also performed with weighted average linkage as the similarity measure. After a relocation procedure (k-means cluster analysis), the resulting solution explained $67 \%$ of the error sums of squares. The ASEDs associated with a match between this cluster solution and the solution of the main analysis using Ward's method ranged from 0.02 to 0.32 with a mean of 0.12 . This represents a good agreement between the solutions and implies that the cluster solution is robust across different clustering methods.

Average absolute scores on quasi absolute scales are reported in Table 3. These average scores validate that the sleep problem cluster (cluster 2) has average levels signifying "insomnia symptoms", the comorbidity cluster (cluster 3) has anxious, depressive as well as insomnia symptoms signifying "clear problems" while the "low mood" (cluster 1) and "distress" (cluster 5) clusters have 
depressive respectively depressive and anxious symptom levels signifying "signs of problems". As can be seen, girls are overrepresented in the clusters characterized by elevated levels of anxiety and/or depression. Furthermore, Table 3 shows that there are significant differences between the clusters in relation to the use of mental control strategies. The low scores cluster scores significantly lower on the use of catastrophic thinking and cognitive avoidance compared to the clusters with elevated symptom levels. The comorbidity cluster scores significantly higher on the use of catastrophic thinking and cognitive avoidance compared to the other clusters. Lastly, Table $\mathbf{3}$ shows that there are significant differences between the clusters in relation to two external criteria related to severity of problems (life satisfaction and school absenteeism). The low scores cluster scores significantly higher on life satisfaction compared to the comorbidity and distress cluster. The comorbidity cluster scores significantly lower on life satisfaction compared to all other clusters. The low scores cluster scores significantly lower on school absenteeism compared to the comorbidity and the low mood cluster. In summary, the differences between the clusters in level of problems on depressive symptoms, anxiety symptoms and sleep problems are confirmed.

Gender did not act as a moderator neither in catastrophic thinking nor in cognitive avoidance (see Table 4). There was a main effect of gender in the use of catastrophic thinking, with girls displaying generally higher levels.

\subsection{The Stability of Depressive Mood, Anxiety and Sleep Problem Clusters across One Year}

A second, similar, cluster procedure was performed cross sectional at wave 2 and the results are displayed in the second column of Figure 2. The first column in this figure is a reordering of Figure 1 and shows the cluster solutions extracted at wave 1 . This display allows for a visual inspection of the structural stability of the cluster constellations across the one year period, as well as for the investigation of typical pathways of change in cluster membership across this time period (arrows signify typical pathways including odds ratios). To assess structural stability, the clusters from wave 1 and 2 are ordered and

Table 3. Descriptive statistics and differences between clusters at wave 1.

\begin{tabular}{|c|c|c|c|c|c|c|}
\hline & $\begin{array}{c}\text { Cluster } 1 \\
\text { Low scores }\end{array}$ & $\begin{array}{c}\text { Cluster } 2 \\
\text { Sleep problems }\end{array}$ & $\begin{array}{c}\text { Cluster } 3 \\
\text { Comorbidity }\end{array}$ & $\begin{array}{c}\text { Cluster } 4 \\
\text { Low mood }\end{array}$ & $\begin{array}{l}\text { Cluster } 5 \\
\text { Distress }\end{array}$ & $\mathrm{H} / \mathrm{F} / \mathrm{Chi}^{2}(\mathrm{df}=4)$ \\
\hline Number ( $\%$ of total) & $155(41 \%)$ & $87(23 \%)$ & $27(7 \%)$ & $75(20 \%)$ & $35(9 \%)$ & \\
\hline Gender (\% girls) & $33 \%$ & $53 \%$ & $85 \%$ & $73 \%$ & $86 \%$ & $64.09^{* * *}$ \\
\hline Depressive symptoms & $0(0)^{\mathrm{a}}$ & $0.8(0.7)^{\mathrm{b}}$ & $2.0(0.5)^{\mathrm{c}}$ & $1.3(0.5)^{\mathrm{c}}$ & $1.5(0.6)^{\mathrm{c}}$ & $282.08^{* * *}$ \\
\hline Anxious symptoms & $0(0.1)^{\mathrm{a}}$ & $0.2(0.4)^{\mathrm{a}}$ & $1.9(0.8)^{b}$ & $0(0)^{\mathrm{a}}$ & $1.3(0.5)^{\mathrm{c}}$ & $301.94^{* * *}$ \\
\hline Sleep problems & $0.8(0.4)^{\mathrm{a}}$ & $2.1(0.3)^{b}$ & $2.4(0.5)^{b}$ & $0.9(0.3)^{\mathrm{a}}$ & $0.9(0.4)^{\mathrm{a}}$ & $300.18^{* * *}$ \\
\hline Catastrophizing & $13.4(4.3)^{\mathrm{a}}$ & $16.5(4.3)^{\mathrm{bc}}$ & $21.0(5.8)^{\mathrm{d}}$ & $16.0(4.5)^{\mathrm{b}}$ & $18.8(4.7)^{\mathrm{cd}}$ & $23.22^{* * *}$ \\
\hline Cognitive avoidance & $9.1(3.2)^{\mathrm{a}}$ & $10.8(3.3)^{\mathrm{ab}}$ & $11.9(3.6)^{\mathrm{b}}$ & $10.1(3.2)^{\mathrm{a}}$ & $11.8(3,0)^{\mathrm{b}}$ & $9.33^{* * *}$ \\
\hline Satisfaction with life & $4.4(0.8)^{\mathrm{a}}$ & $4.0(0.9)^{\mathrm{ab}}$ & $2.5(1.1)^{\mathrm{c}}$ & $4.0(0.8)^{\mathrm{ab}}$ & $3.6(1,1)^{\mathrm{b}}$ & $31.07^{* * *}$ \\
\hline School absenteeism & $2(1-3)^{\mathrm{a}}$ & $2(1-3)^{\mathrm{ab}}$ & $3(1-4)^{b}$ & $2(2-3)^{b}$ & $2(1-3)^{\mathrm{ab}}$ & $21.05^{* * *}$ \\
\hline
\end{tabular}

${ }^{* * *} \mathrm{p}<0.001$ Post hoc tests Games Howell for depressive, anxious symptoms, sleep problems and life satisfaction and Gabriel's for catastrophizing and cognitive avoidance. ${ }^{\mathrm{a}, \mathrm{b}, \mathrm{c}, \text { or } \mathrm{d}}=$ superscripts that highlight significant differences according to post tests (significantly different from the cluster with a different superscript). Depressive symptoms, anxious symptoms and sleep problems are expressed in average levels on quasi absolute scales (see also Table 1). For all variables except school absenteeism Mean (Standard deviation) is displayed. School absenteeism is displayed in median (interquartile range).

Table 4. Does gender moderate the relationship between the use of cognitive strategies and symptom constellation?

\begin{tabular}{|c|c|c|c|c|c|c|c|c|}
\hline & & $\begin{array}{c}\text { Cluster } 1 \\
\text { Low scores }\end{array}$ & $\begin{array}{c}\text { Cluster } 2 \\
\text { Sleep problems }\end{array}$ & $\begin{array}{c}\text { Cluster } 3 \\
\text { Comorbidity }\end{array}$ & $\begin{array}{l}\text { Cluster } 4 \\
\text { Low mood }\end{array}$ & $\begin{array}{l}\text { Cluster } 5 \\
\text { Distress }\end{array}$ & $\mathrm{F}^{\mathrm{a}}(1)$ & $\mathrm{F}^{\mathrm{b}}(4)$ \\
\hline \multirow{2}{*}{ Catastrophizing } & Girls & $14.6(4.8)$ & $17.8(4.3)$ & $21.5(6.2)$ & $16.2(4.1)$ & $18.6(5.1)$ & \multirow{2}{*}{$3.85^{*}$} & \multirow{2}{*}{0.76} \\
\hline & Boys & $12.9(4.0)$ & $15.3(3.9)$ & $18.5(1.3)$ & $14.8(5.7)$ & $18.8(2.9)$ & & \\
\hline \multirow{2}{*}{ Cognitive avoidance } & Girls & $9.3(3.6)$ & $11.4(3.0)$ & $11.6(3.7)$ & $9.9(3.0)$ & $11.7(3.1)$ & \multirow{2}{*}{0.57} & \multirow{2}{*}{1.29} \\
\hline & Boys & $9.1(3.0)$ & $10.2(3.3)$ & $13.3(2.1)$ & $10.1(3.3)$ & $13.2(2.3)$ & & \\
\hline
\end{tabular}

${ }^{*} \mathrm{p}<0.05 . \mathrm{F}^{\mathrm{a}}=\mathrm{F}$-statistic for main effect for gender, $\mathrm{F}^{\mathrm{b}}=\mathrm{F}$-statistic for interaction gender $\mathrm{x}$ cluster. Obs. main effect for cluster is reported in Table 3 . 


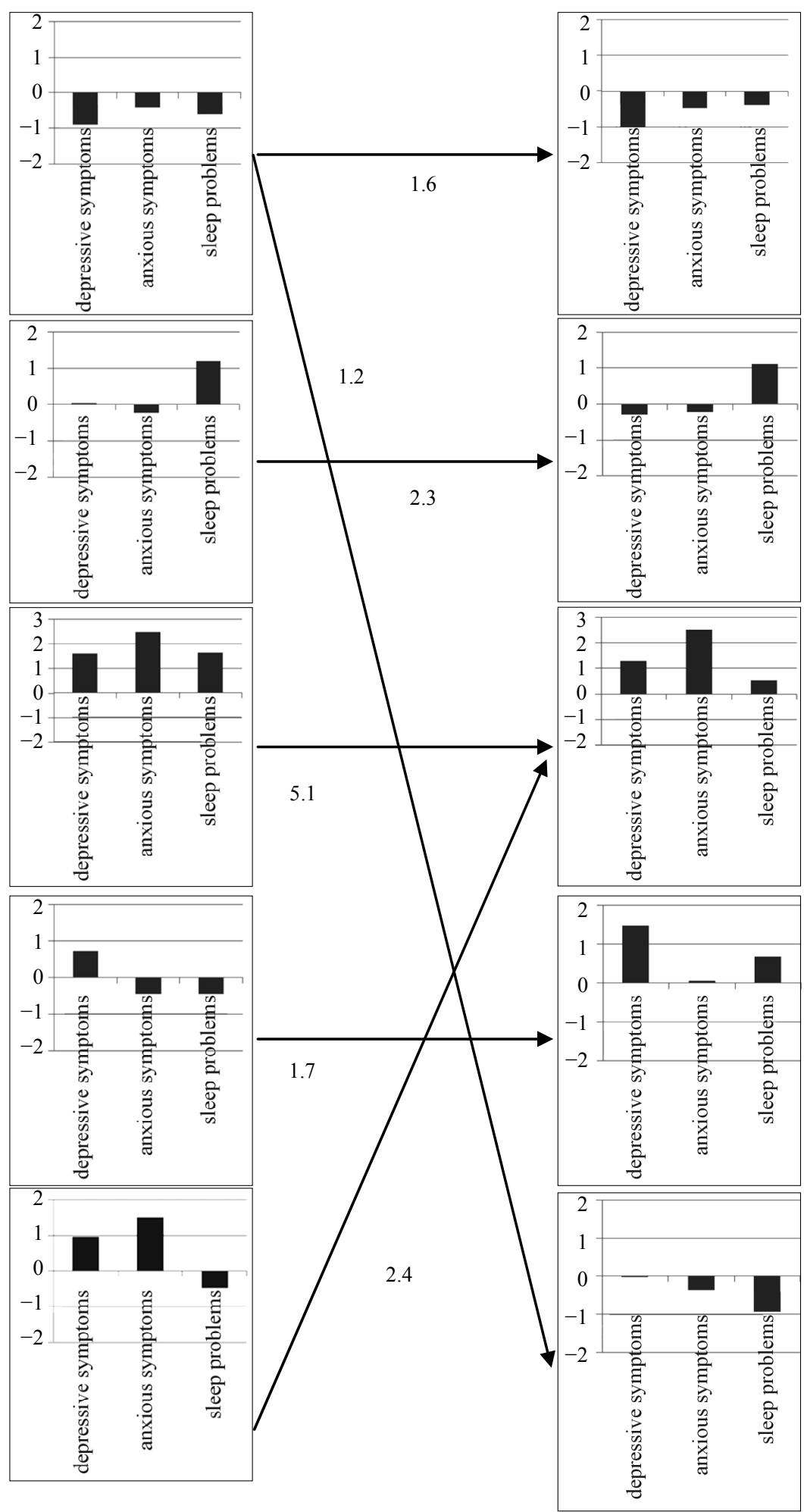

Figure 2. Significant longitudinal streams between baseline clusters (column 1) and assessments at one year follow up (column 2). Note: Graphs depict standardized scores with an area of $\pm 2 \mathrm{z}$-scores on the y-axis. The three variables were profiled in the following order: 1) depressive mood 2) anxiety symptoms and 3) sleep problem. Arrows denote significant developmental pathways from wave 1 to wave 2 . Each arrow is accompanied by an odds ratio (OR) specifying the likelihood of individual stability/change; for all OR $p<0.001$. 
aligned in the columns to reflect their similarity in structure according to the centroid procedure. In this case, the centroid procedure calculates the ASEDs between the centroids of the two cluster solutions at wave 1 and wave 2 that show closest resemblance. As can be seen in the pattern configurations in Figure 2, several, but not all, of the clusters that were extracted at wave 1 reappear in similar configuration at wave 2 . Examined using the centroid procedure this resulted in ASEDs of respectively $0.02 ; 0.06 ; 0.51 ; 0.22$ and 1.22 .

As can be seen in Figure 2 the typical pattern of change across the one year period is one of stability in cluster membership. The comorbidity cluster shows a highest likelihood of individual stability with an odds ratio of 5.1. Noteworthy are the significant pathways of symptom increase from the low mood cluster to low mood in combination with sleep problems at one year follow up (odds ratio 1.7) and from the distress cluster to the cluster with distress including sleep problems (the comorbidity cluster) at one year follow up (odds ratio 2.4).

\subsection{The Relationship between Mental Control Strategies and Symptom Development}

Table 5 shows an exploration of the relationship between trajectories of symptoms change across a one year period and concurrent change in levels of catastrophic thinking and cognitive avoidance. Specifically, the differences in changes in catastrophic thinking and cognitive avoidance across a one year period for subgroups of individuals that have stable levels of (high or low) symptomatology, increased levels of symptomatology and decreased levels of symptomatology were investigated. In order to increase power and facilitate interpretation, clusters at baseline and follow up were simplified and subgrouped into: a problem free group (average scores of below one $\mathrm{z}$ score on all three variables), and a problem group (average $z$ score of $>1$ on one or more of the variables). For this analysis, four trajectories were singled out. Two trajectories of individuals remaining stable ei- ther with (stability symptoms) or without problems with symptoms (stability no symptoms) and two trajectories of individuals changing either from the no problem group to the problem group (increase symptom severity) or vice versa (decrease symptom severity). Table 5 displays the results of a univariate ANOVA investigating differences between trajectories in concurrent changes in catastrophic thinking and cognitive avoidance. As can be seen, a trajectory of decreased symptomatology was accompanied by decreased in catastrophic thinking while a trajectory of increases in symptoms was accompanied by increases in catastrophic thinking as well as cognitive avoidance.

\section{DISCUSSION}

The aim of this study was to examine clusters with different individual profiles of depressive symptoms, symptoms of anxiety and sleep problems. We used longitudinal data from a community sample of adolescents in senior high school and examined the symptom constellations cross-sectional and prospective in relation to the transdiagnostic processes "catastrophic thinking" and "cognitive avoidance" as well as investigated the moderating effect of gender. Finally, we examined the stability of symptom constellations within individuals across a one year time period.

In accordance with our hypothesis, we identified a cluster of adolescents who scored high on depressive symptoms, symptoms of anxiety and sleep problems (the comorbidity cluster). This finding is in line with the comorbidity of mental health problems that has been observed both in general and clinical populations (e.g., [8]). In addition, the adolescents in the comorbidity cluster reported symptom levels at a "clear problem" level on the quasi absolute scales, which are further in line with that if an individual, have multiple problems. It indicates more severe pathology. We further found that adolescents in this comorbidity cluster, together with the adolescents in the distress cluster (elevated levels of depressive and anxiety symptoms), had significantly higher levels of catastrophic thinking compared to adolescents in the other

Table 5. Differences in changes in catastrophic thinking and cognitive avoidance across a one year period for trajectories of stable, increased and decreased symptomatology.

\begin{tabular}{|c|c|c|c|c|c|}
\hline & Stability no symptoms & Decrease symptom severity & Increase symptom severity & Stability symptoms & $\mathrm{F}(3)$ \\
\hline Change in catastrophic thinking & $-0.4(4.6)^{b}$ & $-3.3(4.3)^{\mathrm{a}}$ & $1.8(5.1)^{\mathrm{c}}$ & $-0.5(5.3)^{b}$ & $8.64^{* *}$ \\
\hline Change in cognitive avoidance & $-0.1(3.8)^{\mathrm{a}}$ & $-0.7(3.4)^{\mathrm{a}}$ & $2.1(3.2)^{b}$ & $-0.3(3.3)^{\mathrm{a}}$ & $6.67^{* *}$ \\
\hline
\end{tabular}


clusters. Differences in level of cognitive avoidance between the clusters were most clear between the low scores cluster (significantly lower), and the symptom clusters (significantly higher). Gender did not moderate the relationship between the mental control strategies and cluster membership, but we observed that, independent of cluster membership, adolescent girls used catastrophic thinking to a higher degree than boys. We also found that there was a greater prevalence of adolescent girls in the cluster with a pattern of comorbidity. Hence, those adolescents with some form of comorbid pattern of problems seemed to use a greater degree of mental control strategies than those with a single problem and those with no problems. This is an important finding in the sense that it indicates that it is not only important to be aware of the prevalence and constellation of psychological problems in adolescents, but also of the specific strategies that adolescents use when they try to manage these kind of problems.

The definition and function of catastrophic thinking may give directions in hypothesizing why this mental control strategy could potentially be an important piece of the psychopathology puzzle. As we argued in the introduction, catastrophic thinking can be framed as a thought process under the generic umbrella term repetitive negative thinking (RNT) $([14,15])$. In the worry and rumination literature, it is suggested that RNT with its repetitive, negative and abstract core characteristics may be causally related to decreases in both effective problem solving and emotional processing. At least two pathways are hypothesized. First, this dysfunctional way of regulating negative emotions may increase covert avoidance and in this way maintain long term emotion regulation problems. Second, RNT may hinder a person from effective problem solving and therefore reinforce cognitive and behavioral inflexibility [15]. There are studies that support this view (see for example [14], for a review). Hence, the use of maladaptive mental control strategies such as catastrophic thinking to control unwanted emotional experiences and solve problems may be one explanation of why adolescents who suffer from mental health problems continue to suffer as adults, or develop even more serious problems. Not in the least if catastrophic thinking is used undifferentiated, across many different situations and independent of context, it could make an adolescent less able to adapt to different situations that arise and thus decrease their flexibility further. This could particularly be serious in Western society where a flexible way of thinking and behaving is essential to cope with the increasing demands, for example in preparing for today's working life.

Converging on the above reasoning about why mental health problems in adolescence may risk to be maintained or increased over time, we found empirically that the comorbidity cluster, where adolescents displayed high levels of catastrophic thinking, exhibited stability over a one year period. Compared to the other clusters, the adolescents in this cluster were found to have the highest odds $(\mathrm{OR}=5.1)$ to continue to have comorbid problems the year after. In fact, the general pattern was that those adolescents, who belonged to a cluster with elevated symptoms at time 1 , did not over time move to a cluster with no problems. Instead, it was typical that people either remained in the cluster they belonged to at time 1 , or that they moved to a cluster with more problems. In other words, it was typical that adolescents increased their symptomatology over time and not the other way around. Also, in line with the theoretical idea that if mental control strategies are in fact maintaining ill-health, then in order to reduce symptoms of ill-health, it would take a reduction of the control strategies. Indeed, our results empirically support this hypothesis. We found that a reduction in catastrophic thinking across the one year period was related to a decrease in symptoms. Thus, this may indicate that a reduction in use of this mental control strategy may be important to decrease the mental health symptoms. However, it should be kept in mind that, due to the design of the study with two measurement points, the direction of the relationship between change in symptomatology and mental control strategy cannot be definitely concluded upon.

In close relation to the above and of interest when investigating the development of comorbidity of symptoms, is the temporal order in which the symptoms of anxiety and depression as well as sleep problems arise. We interpret our results to be in line with the more traditional view that sleep problems are a consequence of anxiety and depression and not the other way around (e.g., [32]). In this study, we found that it was a typical developmental pathway (odds higher then chance) that adolescents in the distress cluster across the one year period moved to the comorbidity cluster. In addition, it was also typical that adolescents in the mood cluster (higher levels of depressive symptoms) one year later belonged to a cluster that was characterized by high levels of depressive symptoms and increased sleep problems. Since sleep deprivation has been found to cause problems in emotion regulation and thereby been thought of to increase the risk for enhanced levels of anxiety and depressive symptoms, one might have expected that the results also should have pointed in this direction. However, our results showed no such indication. Instead, our results showed that the only typical pathway for adolescents with singular sleep problems at baseline were likely to continue to belong to the same cluster over the time period of one year. However, it is important to bear in mind that this study is only a snap shot in time and that these processes could have been started in earlier ages. In other words, it is possible that the data in the current study are 
beyond the developmental trajectory where it would be likely to; for example, detect clustering of anxiety and anxiety/sleep problems without mood problems.

Some limitations of the current study should be mentioned. First, we only used self-report measurements that therefore reflect adolescents' subjective perspective only. However, since all questions we ask relate to internalizing problems and mental strategies, it could be argued that that young people themselves should be best suited to answer. Indeed previous research has demonstrated that youths' own reports of internalizing problems are accurate in measuring subjective experiences [33]. Second, in order to create comparable scales we converted our target variables into quasi absolute scales. This rescaling inherently involves variance reduction and therewith may reduce sensitivity for capturing individual variation. The cut-off scores for the scales were, as far as possible, based on reference values from the literature. However, no exactly adapted 4 level cutoffs were available. Therefore, these cut off scores are relative and should be interpreted with caution. Even with cut off values available, these are no absolute standards and the subgroups should therefore be interpreted as broad indications of relations between relative problems levels within individuals only. Third, the data in this study are collected in a senior high school in a small city in a rural area. This means that the results may not automatically generalize to the bigger population senior high school students. Fourth, the measurement instrument of our mental control strategies is newly developed [28]. Therefore the results related to this instrument should be seen as exploratory and further psychometric properties such as construct validity, external validity and reliability need to be confirmed in future studies. Moreover, even though our results are in expected direction we only measured a few mental control strategies and measurements could be improved and broadened. Specifically, measurement of control strategies could be broadened to include behavioral control strategies and a wider range of mental control strategies. Fifth, we used two time points one year apart. It would have been of interest to know more about what happens in between these measurement points. Due to the interval between the two measurement points, changes across time may lack precision. A final point of importance is that cluster analytic methods are based on mathematical models and not on inferential statistics [34]. Therefore, cluster analytic methods are not formulated in the form of a statistical model that considers errors of measurement and sampling errors. It is therefore important to base decisions of inclusion of variables on a sound model and carefully address issues of sample representatives to counterbalance the absence of the possibility to statistically correct for sampling error. This study uses a relatively large sample size in rela- tion to the total population of students from which it was selected. This increases the likelihood that the sample indeed reflects the population from which it was extracted. Further, we have performed additional analyses to establish the robustness of the results by using an alternative clustering method (weighted average linkage), as well as by replicating the analysis on a random subset of the sample. These tests resulted in the same clusters and indicated that the clusters obtained in this study were indeed "real" and did not arise at random owing to the method used or due to "noise" or random data. In addition, the clusters could be meaningfully interpreted and were in line with the scientific literature, showed structural stability across time as well as discriminant validity on indices of transdiagnostic variables and external variables. This further strengthens their reliability and validity. However, replications are needed in samples from different populations, and with variation of measures and methodology used to confirm validity and to be able to draw conclusions of generalizability.

In spite of limitations the current study also has several strengths. First, we use a prospective design with high response rates at each time point (around 80 percent). Second, we have incorporated three highly prevalent mental health problems in adolescence as well as two measures of emotion regulation strategies simultaneously in the same study. This gives us a unique possibility to fill in some of the gaps of knowledge regarding development of mental health problems that exist in the literature. Third, we use a combination of methodologies, variable-oriented and person-oriented, that give us new opportunities to study comorbid mental health problems and development of them within individuals and across time.

\section{CONCLUSION}

To conclude, ill-health symptom patterns differed between adolescents and were either stable over time or increased. Surprisingly, having sleep problems did not increase the risk for enhanced levels of anxiety and depressive symptoms over time; instead anxiety and/or depressive symptoms appeared to be a risk factor for the development of sleep problems. Further, differences between symptom constellations were related to catastrophic thinking and cognitive avoidance. Gender did not act as a moderator but girls, in general, used catastrophic thinking to a higher degree than boys. Thus, our results indicate that the use of maladaptive mental control strategies such as catastrophic thinking and cognitive avoidance may be one explanation of why adolescents suffer from comorbid mental health and continue to suffer. This points to the importance of being aware of the prevalence and constellation of psychological problems in adoles- 
cents, as well as to the specific strategies that adolescents, especially adolescent girls, use when they try to manage these kind of problems.

\section{REFERENCES}

[1] SOU (2006) Ungdomar, stress och psykisk ohälsaAnalyser och förslag till åtgärder. Edita Sverige $\mathrm{AB}$, Stockholm.

[2] Kessler, R.C., Berglund, P., Demler, O., Jin, R., Merikangas, K.R. and Walters, E.E. (2005) Lifetime prevalence and age-of-onset distributions of DSM-IV disorders in the National Comorbidity Survey replication. Archives of General Psychiatry, 62, 593-602.

http://dx.doi.org/10.1001/archpsyc.62.6.593

[3] Ohayon, M.M. (2002) Epidemiology of insomnia: What we know and what we still need to learn. Sleep Medicine Reviews, 6, 97-111.

http://dx.doi.org/10.1053/smrv.2002.0186

[4] Merikangas, K.R., He, J.-P., Burstein, M., Swanson, S.A., Avenevoli, S., Cui, L., Benjet, C., Georgiades, K. and Swendsen, J. (2010) Lifetime prevalence in mental disorders of US adolescents: Results from the National Comorbidity Study-Adolescents Supplement (NCS-A). Journal of the American Academy of Child and Adolescent Psychiatry, 49, 980-989. http://dx.doi.org/10.1016/j.jaac.2010.05.017

[5] Rössner, S. (1998) Childhood obesity and adulthood consequences, Acta Paediatrica, 87, 1-5. http://dx.doi.org/10.1111/j.1651-2227.1998.tb01375.x

[6] Cerdá, M., Sagdeo, A. and Galea, S. (2008) Comorbid forms of psychopathology: Key patterns and future research directions. Epidemiologic Reviews, 30, 155-177. http://dx.doi.org/10.1093/epirev/mxn003

[7] Brooks, T.L., Harris, S.K., Thrall, J.S. and Woods, E.R. (2002) Association of adolescent risk behaviors with mental health symptoms in high school students. Journal of Adolescent Health, 31, 240-246.

http://dx.doi.org/10.1016/S1054-139X(02)00385-3

[8] Harvey, A., Watkins, E., Mansell, W. and Shafran, R. (2004) Cognitive behavioral processes across psychological disorders: A transdiagnostic approach to research and treatment. Oxford University Press Inc., New York.

[9] Wittchen, H.-U., Beesdo, K., Bittner, A. and Goodwin, R.D. (2003) Depressive episodes evidence for a causal role of primary anxiety disorders? European Psychiatry, 18, 384-393.

http://dx.doi.org/10.1016/j.eurpsy.2003.10.001

[10] Jansson, M. and Linton, S.J. (2007) Psychological mechanisms in the maintenance of insomnia: Arousal, distress and sleep-related beliefs. Behaviour Research and Therapy, 45, 511-521. http://dx.doi.org/10.1016/j.brat.2006.04.003

[11] Johnson, E.O., Roth, T. and Breslau, N. (2006) The association of insomnia with anxiety disorders and depression: Exploration of the direction of risk. Journal of Psychiatric Research, 40, 700-708. http://dx.doi.org/10.1016/j.jpsychires.2006.07.008
[12] Taylor, D.J., Lichstein, D.L. and Durrence, H.H. (2003) Insomnia as a health risk factor. Behavioral Sleep Medicine, 1, 227-247. http://dx.doi.org/10.1207/S15402010BSM0104 5

[13] Talbot, L.S., McGlinchey, E.L., Kaplan, K.A., Dahl, R.E. and Harvey, A.G. (2010) Sleep deprivation in adolescents and adults: Changes in affect. Emotion, 10, 831-841. http://dx.doi.org/10.1037/a0020138

[14] Ehring, T. and Watkins, E.R. (2008) Repetitive negative thinking as a transdiagnostic process. International Journal of Cognitive Therapy, 1, 192-205.

http://dx.doi.org/10.1521/ijct.2008.1.3.192

[15] Stöber, J. and Borkovec, T.D. (2002) Reduced concreteness of worry in generalized anxiety disorder: Findings from a therapy study. Cognitive Therapy and Research, 26, 89-96. http://dx.doi.org/10.1023/A:1013845821848

[16] Bergman, L.R. and El-Khouri, B.M. (2002) SLEIPNER-a statistical package for pattern oriented analyses. Department of Psychology, Stockholm University, Stockholm.

[17] Bergman, L.R. (1998) A pattern-oriented approach to studying individual development. Snapshots and processes. In: Cairns, R.B., Bergman, L.R., Kagan, J., Eds., Methods and Models for Studying the Individual, Sage, Thousand Oaks, 83-121.

[18] Von Eye, A. and Bergman, L.R. (2003) Research strategies in developmental psychopathology: Dimensional identity and the preson oriented approach. Development and Psychopathology, 15, 553-580. http://dx.doi.org/10.1017/S0954579403000294

[19] Bergman, L.R., Magnusson, D. and El-Khouri, B. (2003) A person-oriented approach: Methods for today and methods for tomorrow. New Directions for Child and Adolescent Development, 101, 25-38. http://dx.doi.org/10.1002/cd.80

[20] Olsson, G. and von Knorring, A.-L. (1997) Depression among Swedish adolescents measured by the self-rating scale Center for Epidemiology Studies-Depression Child (CES-DC). European Child and Adolescent Psychiatry, 6, 81-87.

[21] Zigmond, A.S. and Snaith, R.P. (1983) The hospital anxiety and depression scale. Acta Psychiatrica Scandinavica, 67, 361-370. http://dx.doi.org/10.1111/j.1600-0447.1983.tb09716.x

[22] Bjelland, I., Dahl, A.A., Haug, T. and Neckelmann, D. (2002) The validity of the hospital anxiety and depression scale. An updated literature review. Journal of Psychosomatic Research, 52, 69-77.

http://dx.doi.org/10.1016/S0022-3999(01)00296-3

[23] Lisspers, J., Nygren, A. and Söderman, E. (1997) Hospital anxiety and depression Scale (HAD): Some psychometric data for a Swedish sample. Acta Psychiatrica Scandinavica, 96, 281-286. http://dx.doi.org/10.1111/j.1600-0447.1997.tb10164.x

[24] Partinen, M. and Gislason, T. (1995) Basic Nordic Sleep Questionnaire (BNSQ): A quantitative measure of subjective sleep complaints. Journal of Sleep Research, 4, 150-155. http://dx.doi.org/10.1111/j.1365-2869.1995.tb00205.x

[25] Liljenberg, B., Almqvist, M., Hetta, J., Roos, B.-E. and 
Ågren, H. (1988) The prevalence of insomnia: The importance of operationally defined criteria. Annals of Clinical Research, 20, 393-398.

[26] Linton, S.J. and Bryngelsson, I.L. (2000) Insomnia and its relationship to work and health in a working-age population. Journal of Occupational Rehabilitation, 10, 169-183. http://dx.doi.org/10.1023/A:1009408204694

[27] American Psychiatric Association (2000) Diagnostic and statistical manual of mental disorders. 4th Edition, American Psychiatric Association, Washington DC.

[28] MacDonald, S., Linton, S.J., and Jansson-Fröjmark, M. (2008) Avoidant safety behaviors and catastrophizing: Shared cognitive behavioral processes and consequences in co-morbid pain and sleep disorders. International Journal of Behavioral Medicine, 15, 201-210. http://dx.doi.org/10.1080/10705500802222675

[29] MacDonald, S., Linton, S.J. and Jansson-Fröjmark, M. (2010) Cognitive vulnerability in the development of concomitant pain and sleep disturbances. British Journal of Health Psychology, 15, 417-434. http://dx.doi.org/10.1348/135910709X468089
[30] Snaith, R.P. (2003) The hospital anxiety and depression scale. Health and Quality of Life Outcomes, 1, 29. http://dx.doi.org/10.1186/1477-7525-1-29

[31] Hair, J., Anderson, R., Tathan, R. and Black, B. (1998) Multivariate data analysis. 5th Edition, Pearson Prentice Hall, Upper Saddle River.

[32] Jansson, M. and Linton, S.J. (2006) The role of anxiety and depression in the development of insomnia: Crosssectional and prospective analyses. Psychology and Health, 21, 383-397. http://dx.doi.org/10.1080/14768320500129015

[33] Trost, K., Biesecker, G., Stattin, H.K. and Kerr, M. (2007) Not wanting parents' involvement: Sign of autonomy or sign of problems? European Journal of Developmental Psychology, 4, 314-331. http://dx.doi.org/10.1080/17405620601008980

[34] Bergman, L.R., Magnusson, D. and El-Khouri, B. (2003) Studying individual development in an interindividual context: A person oriented approach. Lawrence Erlbaum, Mahwah. 